\title{
Planejamento de Programa de Melhoria Abordagem Multimodelo
}

\author{
Cristiane Soares Ramos ${ }^{1}$, Káthia Marçal Oliveira ${ }^{2}$, \\ Ana Regina Cavalcanti da Rocha ${ }^{1}$. \\ ${ }^{1}$ COPPE/UFRJ - Universidade Federal do Rio de Janeiro - Caixa Postal 68511 - CEP \\ 21945-970 - Rio de Janeiro - RJ - Brasil \\ ${ }^{2}$ Laboratoire LAMIH - UMR CNRS 8201, Université de Valenciennes - F-59313 \\ Valenciennes cedex 9, France \\ cristianesramos@unb.br, kathia.oliveira@univ-valenciennes.fr \\ darochadcos.ufrj.br
}

\begin{abstract}
The use of improvement models has been widely adopted to achieve the software organization's business goals. The diversity of services and existing models tends to increase the complexity of the selection of the most appropriated one, especially in multimodels environment. This paper presents a proposal for a strategy to support the planning of process improvement programs. It is based on critical issues that are identified in strategic, tactical and operational levels, and driving the return on investment analysis.
\end{abstract}

Resumo. $O$ uso de modelos de melhoria tem sido uma estratégia amplamente adotada para alcance de objetivos de negócio em organizações de software. A diversidade de serviços prestados e de modelos existentes aumenta a complexidade da seleção do modelo mais adequado, principalmente em ambientes multimodelo. Este trabalho apresenta uma proposta de estratégia para apoiar o planejamento de programas de melhoria de processo em ambiente multimodelo. Ela está fundamentada em Questões Críticas que são identificadas nos níveis estratégico, tático e operacional, direcionam os objetivos do programa de melhoria e a análise do retorno do investimento.

\section{Introdução}

O aumento da competitividade tem despertado o interesse das empresas fornecedoras de serviços de software na busca pela excelência em qualidade. Assim, o estabelecimento de estratégias que apoiem o rompimento das barreiras que as tornem mais competitivas é um fator crítico de sucesso. Embora não haja garantia de que a conformidade com padrões de processo conduzirá a bons produtos, a qualidade do processo poderá influenciar a qualidade do produto que terá a sua qualidade aumentada por meio da melhoria contínua do processo [Naik e Tripathy, 2008]. Desta forma, o uso de normas e de modelos em programa de melhoria de processo tem sido uma estratégia comumente usada pelas empresas que buscam a qualidade dos seus produtos.

Diferentes normas e modelos têm sido criados para apoiar as empresas no alcance dos benefícios provenientes da melhoria de processos. Porém, em função tanto 
da diversidade de modelos disponíveis quanto dos serviços prestados por elas, a seleção do modelo mais adequado tem se mostrado um desafio, pois requer um entendimento profundo dos modelos e também das necessidades de melhoria da empresa, que em muitos casos exige uma abordagem multimodelo, ou seja, implementar mais de um modelo em um mesmo programa de melhoria [Mirna et al., 2011].

As necessidades de melhoria indicam o que é crítico para a empresa e as expectativas com relação aos benefícios esperados do programa de melhoria. A elicitação do que é crítico considera não apenas a participação da alta direção, mas de todos aqueles que são afetados pelas ações de melhoria nos níveis estratégico, tático e operacional. Isso se deve ao fato de que diferentes perfis executam diferentes atividades e, portanto, têm diferentes visões do que precisa ser melhorado e do alcance da melhoria almejada. A participação dos envolvidos em todos os níveis hierárquicos proporciona uma visão holística que considera o todo, as partes e as inter-relações [Ramos et al., 2015].

Os objetivos de um programa de melhoria devem estar alinhados aos objetivos de negócio da organização, de forma que as ações de melhoria causem impacto positivo nos indicadores de negócio [Birkhölzer et al., 2008; Birkholzer et al., 2011]. Portanto, os executivos e também os demais envolvidos em todos os níveis deveriam ser comunicados sobre os ganhos obtidos com esse tipo de programa. Isso torna imprescindível a análise do retorno do investimento (ROI). No entanto, o entendimento das expectativas de benefícios precede a análise do ROI, pois os ganhos obtidos devem também estar alinhados a essas expectativas [Ramos et al., 2012; Ramos et al., 2013].

Neste contexto, este trabalho descreve uma proposta para planejamento de programas de melhoria de processos com o propósito de identificar os problemas críticos da empresa e os benefícios que ela espera alcançar. Eles apoiam a definição dos objetivos do programa de melhoria e a análise do alcance das expectativas de benefícios.

$\mathrm{Na}$ sequência deste artigo, a seção 2 apresenta uma breve revisão de literatura. A Seção 3 a descrição da Estratégia com foco no planejamento do programa de melhoria. A Seção 4 mostra a aplicação da Estratégia e as lições aprendidas. E, finalmente, a Seção 5 as considerações finais.

\section{Trabalhos relacionados}

Programas de melhoria de processo são concebidos para projetar e definir melhorias nos processos, de forma que eles contribuam para que a empresa alcance seus objetivos de negócios [Rico, 2004; Birkhölzer et al., 2008; Guzmán et al., 2010; Birkholzer et al., 2011]. A continuidade dos programas de melhoria tem sido um desafio, pois a falta de clareza quanto aos benefícios obtidos enfraquece o comprometimento da alta direção [Solingen, 2004], podendo inclusive chegar a comprometer investimentos financeiros. Desta forma, a análise do ROI é um fator crítico de sucesso em programas de melhoria [Ferreira et al., 2008]. Apesar disso, é comum as empresas iniciarem esses programas sem antes definirem suas expectativas de benefícios e, ao final, não avaliarem se elas foram alcançadas.

Modelos de qualidade vêm sendo estrategicamente utilizados em empresas para apoiá-las no alcance dos seus objetivos de negócio. Porém, empresas com cenários 
complexos, em que os serviços prestados ultrapassam o escopo do desenvolvimento de software podem existir. Como exemplo: (i) empresas que, além de desenvolverem produtos de software, oferecem serviços de help desk ou service desk; e (ii) empresas que contratam em parte, ou no seu total, o desenvolvimento de software por meio de outsourcing.

A implementação simultânea de múltiplos modelos permite o tratamento de diferentes pontos de melhoria na empresa de uma forma mais adequada. No entanto, o programa de melhoria, deve ser estruturado no sentido de viabilizar a harmonização dos modelos evitando custos desnecessários, desperdício de recursos e retrabalho. Neste cenário surgiu o conceito de ambiente multimodelo como resultado de um esforço das empresas para integrar modelos em programas de melhoria de processo [Siviy et al., 2008a]. Porém, o seu uso requer o entendimento e a interpretação do quão diferentes são os modelos e como eles se correlacionam. Isso torna uma implementação multimodelo uma tarefa complexa. Logo, o uso de uma estratégia que harmonize e combine esses modelos é um fator crítico de sucesso [Pardo et al., 2012].

As iniciativas multimodelo, podem ser classificadas em [Kelemen, 2013]: Mapeamento; Integração e Harmonização. $\mathrm{Na}$ ontologia proposta por Pardo et al. (2012), elas são assim descritas: (1) Mapeamento: técnica de comparação que vai além da identificação das semelhanças e diferenças dos modelos que são comparados. Ela requer a análise dos elementos dos modelos envolvidos no mapeamento (ex.: atividades, produtos de trabalho, tarefas, etc.) e não apenas dos processos ou grupo de processos; (2) Integração: ação para unir ou mesclar dois ou mais modelos. Um dos maiores objetivos de iniciativas de harmonização é apoiar a integração de múltiplos modelos com um framework de integração. Permite que diferentes modelos sejam harmonizados quando a colaboração entre eles é necessária em uma organização; e (3) Harmonização: Desenvolvimento de uma solução adequada que permita que os objetivos da organização sejam satisfeitos.

Desta forma, a harmonização implica em definir soluções que apoiem a empresa [Siviy et al., 2008a]: (1) no entendimento de quais modelos ajudam-na a alcançar os seus objetivos de negócio; (2) no entendimento das diferenças e sobreposições que caracterizam esses modelos; (3) na criação de um processo organizacional focado na missão da empresa e na incorporação de características e conteúdo de todos os modelos escolhidos; (4) na implementação de uma solução multimodelo e na medição dos resultados. Nessa linha podem ser citados os trabalhos de Mirna et al. (2011), e o PRIME (Process Improvement in Multimodel Environments) [Siviy et al., 2008b]. Esses dois estudos medem os resultados e avaliam o alcance dos objetivos de negócio. Salviano (2009) define uma abordagem orientada a perfil de capacidade de processo. Outros exemplos de abordagens multimodelo são encontrados [Pardo et al., 2011].

Alguns benefícios que justificam o uso de abordagens multimodelo são [Siviy et al., 2008b]: focar no negócio ao invés de focar no modelo; mudança cultural em relação à implantação de processos na empresa; sistema de medição; abordagem organizacional robusta e efetiva em um programa de longo prazo. No entanto, ela apresenta também alguns desafios [Andelfinger et al., 2006; Ibrahim, 2008]: obter o comprometimento da alta direção; determinar a estratégia organizacional; integrar e coordenar treinamentos; 
integrar medições que não são foco do modelo adotado; conhecer as diferenças e similaridades entre os diferentes modelos adotados no programa de melhoria.

Neste contexto, faz-se necessária uma abordagem para apoiar o planejamento de programas de melhoria de processos harmonizando os diferentes modelos selecionados visando atender tanto às questões críticas de processo quanto às expectativas de benefícios da empresa. E que, ao final de cada ciclo, promova a análise dos ganhos obtidos com base nas expectativas iniciais da empresa.

\section{Uma Estratégia para Planejamento de Programa de Melhoria}

Assim como a abordagem IDEAL (Initiating, Diagnosing, Establishing, Acting \& Learning) [Mcfeeley, 1996], a Estratégia proposta nesse trabalho é iterativa. Ela considera que um programa de melhoria deve ser executado em ciclos, propiciando avaliar mais rapidamente os ganhos que vêm sendo obtidos com as melhorias implementadas a cada ciclo e provendo respostas rápidas às possíveis mudanças de expectativas quanto aos ganhos esperados. Os resultados obtidos são insumos para os próximos ciclos.

A Estratégia é composta por quatro grandes etapas, conforme apresentado na Figura 1. A premissa principal é que as expectativas de benefícios sejam claramente identificadas para então guiarem a definição dos objetivos do programa de melhoria. Assim, ao final de cada ciclo de melhoria, avalia-se o alcance das expectativas iniciais. Sendo assim, a Etapa 1 tem como objetivo o entendimento das características da empresa quanto aos serviços prestados, iniciativas de melhoria atuais, passadas, etc. Em seguida, identifica-se o que é crítico para a empresa e as expectativas de benefícios, denominadas Questões Críticas (QC). Isso é feito sob a perspectiva dos envolvidos no programa de melhoria nos níveis estratégico, tático e operacional.

As QC são identificadas com o uso de Questionários em que os colaboradores indicam, para cada questão, o grau de importância e o grau de percepção. Neste caso, a importância indica a relevância da questão na visão do respondente, enquanto a percepção indica o quanto o item abordado é executado na prática da empresa. As questões classificadas com alta importância e baixa percepção são consideradas críticas, ou seja, indicam quais aspectos devem ser melhorados na empresa e, portanto, priorizados no planejamento de um programa de melhoria. Elas representam o estímulo para a mudança.

Cada uma das questões dos questionários está associada a processos e consequentemente a modelos de melhoria. Há dois tipos de questões. As Questões de Processo que indicam quais processos necessitam de melhoria e as Questões de Benefícios que indicam quais os benefícios esperados do programa de melhoria. $O$ processo de criação dos questionários está baseado na proposta de Pasquali (1999), com a execução dos procedimentos teóricos (fundamentação teórica e validação semântica das questões) e dos procedimentos experimentais (planejamento e aplicação do questionário), cujo detalhamento pode ser obtido em [Ramos, 2015].

O embasamento teórico dos questionários está apoiado em duas fontes principais: (1) o mapeamento sistemático da literatura [Ramos et al., 2013] em que foram mapeados benefícios de Programas de Melhoria de Processo de software; e (2) 
modelos de melhoria no contexto de Software (MPS-SW [Softex, 2012b] e CMMI-DEV [Sei, 2010a]), Serviços (MPS-SV [Softex, 2012a] e CMMI-SVC [Sei, 2010b]) e Testes (MPT.Br [Softex-Recife, 2011]). Esses modelos foram escolhidos por serem amplamente usados nas empresas brasileiras [Neto et al., 2011]. Eles estão baseados também na proposta de Xexéo (2001) que faz uma adaptação do SERVQUAL [Parasuraman et al., 1988] e considera que todas as partes interessadas devem participar do processo de identificação das prioridades de um programa de qualidade. Essa é mais uma das premissas da nossa Estratégia.

Na Etapa 2, o planejamento do programa de melhoria é realizado com base nas QC. Neste planejamento os objetivos, os ciclos do programa de melhoria, o escopo de cada ciclo e as medidas necessárias para avaliação do alcance das expectativas de benefícios são definidos. Além disso, são incorporados aspectos que propiciam a harmonização de modelos para ambientes multimodelo. Na Etapa 3, é executado o planejamento feito na Etapa 2 e a coleta das medidas, gerando insumos para que na Etapa 4 sejam analisados os ganhos obtidos no ciclo de melhoria em função das expectativas iniciais.

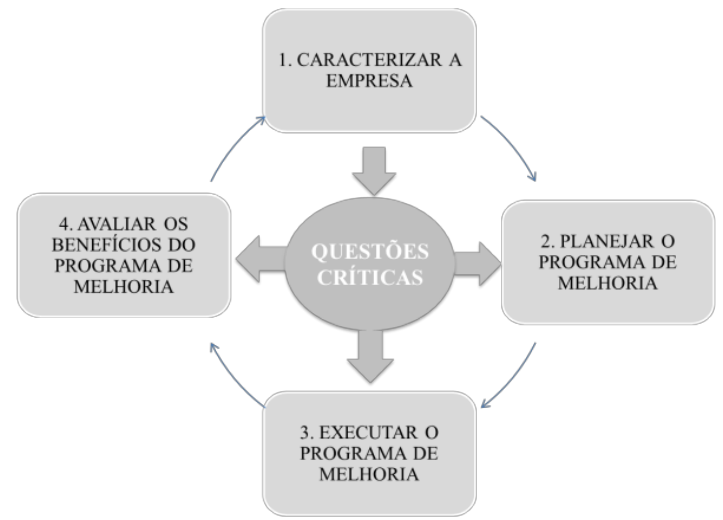

Figura 1. Estratégia para análise de benefícios em Programas de Melhoria

A seguir são descritas as atividades da Etapa 2:

Atividade 1 - Identificar processos relevantes: O primeiro passo dessa atividade é identificar os modelos candidatos e os processos que atendam às necessidades de melhoria da empresa a partir das QC. Além disso, são consideradas as restrições de negócio, o histórico de iniciativas de melhoria da empresa e os modelos que já estejam em uso. É necessário que se entenda o nível de sobreposição (parcial ou total) entre os modelos candidatos e seus processos, e também as suas diferenças quanto: ao propósito; ao conteúdo; às práticas; e à arquitetura. Essa análise é importante, pois esses modelos serão harmonizados quando da definição ou melhoria dos processos da empresa. Esse passo é crucial para minimizar custos e esforços do programa de melhoria.

Atividade 2 - Definir objetivos do Programa de Melhoria: Os objetivos do programa de melhoria devem estar alinhados às QC, pois elas foram apontadas pelos colaboradores como aspectos relevantes a serem melhorados com a implantação do programa de melhoria. Logo, devem efetivamente ser consideradas ao definir os objetivos do programa. 
Atividade 3 - Definir e Selecionar alternativas de solução: O primeiro passo dessa atividade é a Definição das Alternativas de Solução, ou seja, definição dos cenários que possam favorecer o alcance dos objetivos do programa de melhoria, e consequentemente o tratamento das QC. O ponto de partida é indicar o tipo de solução a ser adotada: (1) Solução monomodelo - implementa apenas um modelo de melhoria; ou (2) Solução multimodelo - implementa mais de um modelo de melhoria.

Em seguida, para cada tipo de solução, é definida a forma de implementação: (1) Representação por estágios - implementação de um conjunto pré-definido de processos de um determinado nível de maturidade; (2) Representação contínua - escolha de um ou mais processos para melhoria; ou (3) Representação Híbrida - uso da representação por estágio com a inclusão de outros processos que a empresa queira melhorar, ou seja, implementar um determinado nível de maturidade e incluir processos de outros níveis ou até mesmo de outros modelos de melhoria.

Os colaboradores indicam um conjunto de QC que nem sempre podem ser tratadas de uma única vez, por isso precisam ser divididas em ciclos de melhoria. Neste caso, são priorizadas pela alta direção, ou seu representante. Esta decisão pode ser apoiada pelo Índice de Relevância do Processo (IRP), um indicador que considera a importância e a percepção relativas dos processos no conjunto de QC. Ele é calculado pela fórmula: (Média da importância relativa da QC dividido pela Média da Percepção relativa $d a Q C$ ). Como cada questão está associada a um processo, esse valor é atribuído ao processo.

Para a Seleção das Alternativas de Solução foi adotada uma adaptação da técnica PICK chart do Lean Six Sigma [Mike et al., 2004]. Ela apoia a empresa na priorização das soluções e ideias, separando-as em quatro categorias: (P) Possible (Possível) - baixo retorno e fácil de implementar; (I) Implement (Implementar) - Alto retorno e fácil de implementar; (C) Challenge (Desafio) - Alto retorno e difícil de implementar; e (K) Kill (Descartar) - Baixo retorno e Difícil de Implementar. Desta forma, para classificar as alternativas de solução no PICK chart, deve-se analisar a Facilidade de implementação - está relacionada com o esforço demandado para implementar a solução; e analisar também o tipo de Retorno esperado. A solução a ser selecionada é a que for classificada no quadrante "Implementar".

Diferentes critérios podem apoiar a análise sobre a "facilidade de implementação" e sobre o "retorno". No que se refere à facilidade de Implementação, podemos citar: necessidades de treinamento; contratação de consultoria; esforço de implementação; desenvolvimento ou compra de ferramentas de apoio; disponibilidade de pessoal; dentre outros. No que se refere ao retorno, pode-se considerar: o grau de cobertura das QC; o atendimento às restrições de negócio; dentre outros que podem ser sugeridos pela própria empresa.

Atividade 4 - Definir medidas de sucesso do Programa de Melhoria: Finalmente, com base nas QC de benefícios, são determinadas as medidas a serem coletadas a fim de apoiar a avaliação dos ganhos obtidos com o programa de melhoria. As medidas serão selecionadas a partir de um Catálogo de Medidas para avaliação do ROI em melhoria de processo. 


\section{APLICAÇ̃̃o PRÁTICA dA ESTRATÉGIA}

A Estratégia, até o momento, foi aplicada em duas empresas. Na subseção 4.1 são apresentados os resultados obtidos na Empresa A, e na subseção 4.2 um resumo dos resultados da Empresa B. Na subseção 4.3 discutem-se as lições aprendidas e oportunidades de melhoria da Estratégia.

\subsection{EMPRESA A}

\section{ETAPA 1 - Caracterização da empresa e identificação das questões críticas}

A Empresa A que é fornecedora de serviços de Tecnologia da Informação (TI), atua em todo o Brasil empregando mais de 10 mil colaboradores. Nos últimos anos ela teve um faturamento acima de 300 milhões de dólares em linhas de negócio como: desenvolvimento e manutenção de software; call-center; outsourcing; comercialização de produtos, e outros. A Estratégia foi aplicada no contexto da linha de negócio Desenvolvimento e Manutenção de Software (neste trabalho ela é referenciada como DMS). Ela tem mais de 150 colaboradores e vários clientes, principalmente no governo. É avaliada no nível 3 de maturidade do CMMI-DEV.

O conjunto de QC é o produto de entrada para o planejamento do programa de melhoria. Para elicitá-las todos os colaboradores foram convidados a responder os questionários. Obtivemos como resposta uma cobertura de 100\% dos perfis com $13 \%$ do contingente de colaboradores que atuam na unidade: (1) Equipe técnica (Data Base Administrators (DBA); programadores; analistas de requisitos; arquitetos de software; testadores); (2) Gerentes de projeto; (3) Apoio a processos (analistas de métricas; analistas de qualidade); (4) Alta direção (gerente da unidade). O tempo de atuação dos colaboradores (respondentes) na empresa varia de 2 meses a 8 anos.

Como proposto por Xexeo (2001), a partir das respostas dos questionários, para cada perfil, as questões foram classificadas nos quadrantes: (Q1) - Alta importância e Alta percepção; (Q2) Alta importância e Baixa percepção; (Q3) Baixa Importância e Baixa Percepção; (Q4) Baixa importância e Alta percepção. O Quadro 1 mostra exemplos de questões de processo para o perfil "Gerente de Projeto".

Quadro 1 - Exemplos Questões de Processo por Quadrante - Perfil: - Gerente de proj.

\section{Q2 - alta importância e baixa percepção $\quad$ Q1 - alta importância e alta percepção}

- Uso de critérios para aceitação de um novo projeto - Definição e padronização dos processos da ou serviço

- Melhoria contínua dos processos

- Capacitação dos colaboradores

- Mitigação e contingência p/os riscos dos projetos

Q3 - baixa importância e baixa percepção

- Troca de conhecimento entre os colaboradores

- Gerência de reuso de software

-Desenvolvimento de software visando futuro reuso. empresa

- Uso de templates para padronização da documentação dos projetos

- Uso de técnicas de estimativas

Q4 - baixa importância e alta percepção

- Planejamento da carteira de projetos e serviços

- Uso de medidas para apoio à tomada de

decisões

- Desenvolvimento dos requisitos

As QC são aquelas classificadas no quadrante Q2 (alta importância e baixa percepção). Desta forma, no Quadro 2 estão listadas todas as QC apontadas por todos os perfis, mesmo aquelas que não são coincidentes. As questões de Processo indicam aspectos a serem melhorados nos processos de software da DMS, e as Questões de 
Benefícios indicam os benefícios esperados do programa de melhoria. Observa-se que mesmo a empresa já tendo sido avaliada no nível de maturidade (ML) 3 do CMMI$\mathrm{DEV}$, foram identificadas QC relacionadas a processos do nível 2 e também do nível 3.

Quadro 2 - Questões Críticas de Processo e de Benefícios (todos os perfis)

\begin{tabular}{|c|c|}
\hline QUESTÕES CRÍTICAS DE PROCESSO & QUESTÕES CRÍTICAS DE BENEI \\
\hline $\begin{array}{l}\text { Q03 Armazenamento organizado e controlado dos } \\
\text { documentos dos projetos e processos } \\
\text { Q04 Verificar se os colaboradores seguem os } \\
\text { processos e utilizam os templates dos } \\
\text { documentos. } \\
\text { Q05 Uso de critérios para aceitação de um novo } \\
\text { projeto ou serviço } \\
\text { Q06 Análise periódica da viabilidade de } \\
\text { continuidade dos projetos e serviços } \\
\text { Q07 Uso de medidas para avaliação do alcance dos } \\
\text { objetivos da empresa } \\
\text { Q08 Uso de medidas para apoio à tomada de } \\
\text { decisões } \\
\text { Q09 Melhoria contínua dos processos } \\
\text { Q11 Capacitação dos colaboradores } \\
\text { Q12 Troca de conhecim. entre os colaboradores } \\
\text { Q13 Uso de critérios para tomada de decisões } \\
\text { críticas. } \\
\text { Q14 Mitigação e contingência para os riscos dos } \\
\text { projetos. } \\
\text { Q15 Planejam. da carteira de projetos e serviços } \\
\text { SW01 Gerência de reuso de software } \\
\text { SW02 Desenvolv. de software visando futuro reuso. } \\
\text { SW06 Execução de revisão por pares } \\
\text { SW07 Assegurar homologação satisfatória do } \\
\text { produto. }\end{array}$ & $\begin{array}{l}\text { B02 Melhoria no planejamento do projeto } \\
\text { B04 Melhoria da precisão das estimativas } \\
\text { B05 Redução de horas-extras } \\
\text { B06 Aumento da produtividade } \\
\text { B07 Redução de retrabalho } \\
\text { B08 Redução de custos } \\
\text { B09 Melhoria da qualidade de vida dos } \\
\text { colaboradores. } \\
\text { B10 Aumento da satisfação dos colaboradores } \\
\text { B11 Aumento do tamanho médio dos projetos } \\
\text { B12 Aumento do número de projetos no Brasil } \\
\text { B13 Aumento do número de projeto exterior } \\
\text { B14 Aumento do número de clientes no Brasil } \\
\text { B15 Aumento do número de clientes no exterior } \\
\text { B16 Aumento da satisfação do cliente } \\
\text { B18 Melhoria do time to Market } \\
\text { B20 Núm. de colab. adequados às demandas } \\
\text { B21 Gestão da comunicação } \\
\text { B22 Cumprimento de prazo } \\
\text { B23 Redução de esforço } \\
\text { B24 Melhoria no monitoramento do projeto } \\
\text { B25 Aumento da fatia de mercado } \\
\text { B26 Aumento da competitividade da empresa } \\
\text { B27 Recompensa para os colaboradores } \\
\text { B28 Redução de multas } \\
\text { B29 Aumento do faturamento }\end{array}$ \\
\hline
\end{tabular}

Os tópicos a seguir descrevem como as questões críticas foram usadas no planejamento do programa de melhoria.

\section{ETAPA 2 - Planejamento do Programa de Melhoria}

Atividade 1 - Identificar o(s) modelo(s) candidato(s) e os processos relevantes: Cada QC está associada a um processo, e este por sua vez está associado a um modelo de melhoria. Sendo assim, as QC críticas conduzem à seleção dos modelos e dos processos. Neste caso, foram então selecionados os modelos CMMI-DEV e MPS-SW. Porém, foram considerados também os elementos-chave apresentados no Quadro 3.

Quadro 3 - Elementos-chave usados para apoiar a seleção dos modelos candidatos

CMMI-DEV

- Restrição de negócio: A empresa tem contratos que exigem a manutenção do CMMI-DEV no ML3

- Modelo em uso na empresa: definidos segundo o modelo CMMI-DEV

- Histórico de iniciativas de melhoria: esse modelo vem sendo usado na empresa há alguns anos.

- Negócios no exterior: Expectativa de conquistar clientes e projetos no exterior (B13 e B15) MPS-SW

- Escopo: tratamento de QC que não são cobertas pelo CMMI-DEV: Ger. de Portfólio de Projetos (Q05; Q06; Q15); Gerência de conhecimento (Q12); e Reuso de software (SW01 e SW02). 
Uma vez estabelecida a necessidade de implementação dos dois modelos candidatos, foi preciso entender como esses modelos se relacionam. Sendo assim, foi usado o mapeamento do MPS-SW com o CMMI-DEV, identificando as sobreposições e diferenças entre eles [Softex, 2012c]. Como apresentado na Figura 2, os processos cobertos nos dois modelos estão destacados em cinza. Os demais são cobertos apenas pelo modelo MPS-SW (GRU; DRU; GPP; e parte de GRH que tem sobreposição com o processo Treinamento do CMMI-DEV, porém GRH possui práticas adicionais voltadas para gerência do conhecimento). A Figura 2 apresenta também a rastreabilidade entre os processos e as questões críticas.

\begin{tabular}{|c|c|c|c|c|c|c|c|}
\hline $\begin{array}{l}\mathrm{ML} \\
3 \mathrm{C}\end{array}$ & \multicolumn{2}{|c|}{ GRI - Gerência de Riscos - Q14 } & \multicolumn{3}{|c|}{ GDE - Gerência de Decisões - Q13 } & \multicolumn{2}{|c|}{$\begin{array}{l}\text { DRU - Desenv olvimento para } \\
\text { reutilização } \mathrm{SW}^{2} 02\end{array}$} \\
\hline D & $\begin{array}{c}\text { DRE - } \\
\text { Desenvolvim ento de } \\
\text { Requisitos }\end{array}$ & \multicolumn{2}{|c|}{$\begin{array}{l}\text { PCP - Projeto e } \\
\text { Construção do } \\
\text { Produto }\end{array}$} & $\begin{array}{c}\text { ITP - Integração do } \\
\text { Produto }\end{array}$ & \multicolumn{2}{|c|}{$\begin{array}{c}\text { VER - Verifica ção } \\
\text { SW06 }\end{array}$} & $\begin{array}{c}\text { VAL - Validação } \\
\text { SWV07 }\end{array}$ \\
\hline $\mathrm{E}$ & \multicolumn{2}{|c|}{$\begin{array}{l}\text { GRH - Gerência de Recursos } \\
\text { Humanos Q11/ Q12 }\end{array}$} & $\begin{array}{r}\text { DFP - } \\
\text { Processo }\end{array}$ & $\begin{array}{l}\text { nição do } \\
\text { inizacional }\end{array}$ & \multicolumn{2}{|c|}{$\begin{array}{c}\text { AMP - Ava lia ção e } \\
\text { Melho ria do Processo } \\
\text { Organizacional-Q09 }\end{array}$} & $\begin{array}{l}\text { GRU - Gerência de } \\
\text { reutilização-SW01 }\end{array}$ \\
\hline \multirow{2}{*}{$\begin{array}{l}\mathrm{ML} \\
2 \mathrm{~F} \\
\mathrm{G}\end{array}$} & $\begin{array}{c}\text { MED - Medição } \\
\text { Q07/Q08 }\end{array}$ & \multicolumn{2}{|c|}{$\begin{array}{c}\text { GQA - Garantia da } \\
\text { Qualidade Q04 }\end{array}$} & $\begin{array}{l}\text { GCO-Gerência de } \\
\text { Config Q Q03 }\end{array}$ & $\begin{array}{l}\text { AQU - } \\
\text { Aqui siçãa }\end{array}$ & \multicolumn{2}{|c|}{$\begin{array}{l}\text { GPP-Gerência de Portfólio } \\
\text { de Projetos Q05 / Q06/ Q15 }\end{array}$} \\
\hline & \multicolumn{4}{|c|}{ GPR - Gerência de Projetos } & \multicolumn{3}{|c|}{ GRE -Gerência de Requisitos } \\
\hline
\end{tabular}

Figura 2. Rastreabilidade dos processos do MPS-SW com as questões críticas

Atividade 2 - Definir objetivos do Programa de Melhoria: Em reunião com o gerente de qualidade da DMS foram definidos seguintes objetivos para o programa de melhoria: Melhoria da satisfação do cliente; Melhoria da Qualidade do produto; Redução de custos de desenvolvimento; Melhoria da capacitação dos colaboradores.

Atividade 3 - Definir e Selecionar alternativas de solução: Ao definir as alternativas de solução, primeiramente deve-se indicar o tipo de solução: monomodelo ou multimodelo. Como mostrado na atividade 1, a avaliação das QC indica a necessidade de uma solução multimodelo, pois apenas um dos modelos CMMI-DEV ou MPS-SW não atenderia às $\mathrm{QC}$ e também à restrições de negócio da empresa. Por exemplo, o CMMI-DEV cobre apenas 63\% das QC e o MPS-SW não atende aos contratos de clientes que exigem a implementação do ML3 do CMMI-DEV. O próximo passo é definir a forma de implementação: Representação por estágio, Representação contínua ou Representação híbrida. Então, foram propostas duas alternativas de solução multimodelo:

- Alternativa A - Está baseada em uma abordagem multimodelo de representação contínua. A priorização dos processos é feita com base no IRP. Desta forma, foram planejados 4 ciclos de melhoria (Quadro 4). No $1^{\circ}$. ciclo são priorizados os processos GPP, MED e GRI, pois são os que possuem os três maiores IRP $(21,17 \%$; $10,07 \%$; e $7,20 \%$ respectivamente). O $2^{\circ}$. e $3^{\circ}$. ciclos implementam os demais processos que também foram priorizados segundo o IRP (com exceção do processo DRU que foi colocado no $3^{\circ}$. Ciclo para que sua implementação ocorra após a do GRU). No $4^{\circ}$. Ciclo são implementados os processos do ML2 e ML3. Esta alternativa tem como principal vantagem o fato de serem priorizados no $1^{\circ}$. Ciclo de melhoria os processos que são mais relevantes para os colaboradores. A principal desvantagem é o fato de requerer uma implementação mais complexa, pois a representação contínua pode exigir um pouco mais de gestão por parte da equipe de qualidade, além de exigir a avaliação da correlação entre os processos e atributos de processo. 
- Alternativa B - também está baseada em uma abordagem multimodelo, porém com representação híbrida. Em cada nível de maturidade do CMMI-DEV são agregados os processos do MPS-SW do nível de maturidade correspondente. Como mostrado no Quadro 4, foram planejados 3 ciclos de melhoria. Os processos são priorizados em função do nível de maturidade. Sendo que DRU foi colocado no $3^{\circ}$. Ciclo para que seja implementado após GRU. Esta alternativa tem como principal vantagem a inclusão gradativa dos processos do MPS-SW. Como desvantagem, o aumento da complexidade de implementação por agregar mais processos aos níveis de maturidade do CMMI-DEV.

Nas duas alternativas de solução, para distribuir os processos nos ciclos de melhoria, considerou-se ainda que independentemente da estruturação dos ciclos de melhoria, os processos GPR e GRE devem ser incluídos no $1^{\circ}$. ciclo, uma vez que eles geram insumos relevantes e algumas vezes requeridos para a execução dos demais processos. O Quadro 4 apresenta um resumo dos ciclos de melhoria para as duas alternativas de solução, o escopo de cada ciclo e as respectivas QC.

Quadro 4 - Resumo das alternativas de solução

\begin{tabular}{|c|c|c|}
\hline & Alternativa A & Alternativa B \\
\hline $\mathbf{1}^{\mathrm{o}}$ & $\begin{array}{l}\text { Processo: GPP MED GRI GPR GRE } \\
\text { QC: Q05, Q06, Q15, Q07, Q08, Q14 }\end{array}$ & $\begin{array}{l}\text { Processo: CMMI-DEV ML2 + GPP } \\
\text { QC: Q07 Q08 Q04 Q03 Q05 Q06 Q15 }\end{array}$ \\
\hline $2^{\circ}$ & $\begin{array}{l}\text { Processo: GRU GQA GCO GDE } \\
\text { QC: SW01, Q04, Q03, Q13 }\end{array}$ & $\begin{array}{l}\text { Processo: CMMI-DEV ML3 + GRH + GRU } \\
\text { QC: Q11, Q09, SW01, Q11, Q12, SW06, SW07, } \\
\text { Q14, Q13 }\end{array}$ \\
\hline $3^{\circ}$. & $\begin{array}{l}\text { Processo: AMP GRH VER VAL DRU } \\
\text { QC: Q09, Q11, Q12, SW06, SW07, SW02 }\end{array}$ & $\begin{array}{l}\text { Processo: DRU } \\
\text { QC: SW02 }\end{array}$ \\
\hline $4^{\mathbf{0}}$ & Processos restantes do ML2 e ML3 & --- \\
\hline
\end{tabular}

Após definir as alternativas de solução, o passo seguinte foi analisá-las quanto à Facilidade de Implementação e Retorno. O resultado é apresentado no Quadro 5.

Quadro 5 - Avaliação das Alternativas de Solução

\begin{tabular}{|c|c|c|}
\hline & Facilidade Implementação & Retorno \\
\hline $\mathbf{A}$ & $\begin{array}{l}\text { Difícil } \\
\text { A ordem de implementação dos processos é } \\
\text { orientada pela relevância dos processos e não } \\
\text { pelo nível de maturidade. Isso dificulta a } \\
\text { interconexão, entre processos, exigida pelas } \\
\text { práticas genéricas / atributos de processo. }\end{array}$ & $\begin{array}{l}\text { Alto } \\
\text { - Cobertura de 100\% das QC de processo } \\
\text { - Implementação dos processos com maior } \\
\text { relevância nos primeiros ciclos de melhoria } \\
\text { - Avaliação conjunta dos modelos e aumento } \\
\text { da visibilidade da empresa } \\
\text { - Atendimento das restrições de negócio }\end{array}$ \\
\hline B & $\begin{array}{l}\text { Fácil } \\
\text { A ordem de implementação dos processos é } \\
\text { orientada pelos níveis de maturidade e os } \\
\text { processos do MPS-SW são agregados } \\
\text { gradativamente aos níveis de maturidade do } \\
\text { CMMI-DEV a cada novo ciclo de melhoria. }\end{array}$ & $\begin{array}{l}\text { Alto } \\
\text { - Cobertura de } 100 \% \text { das QC de processo } \\
\text { - Avaliação conjunta dos modelos e aumento } \\
\text { da visibilidade da empresa } \\
\text { - Atendimento das restrições de negócio }\end{array}$ \\
\hline
\end{tabular}

Em seguida, as alternativas de solução foram classificadas nos quadrantes do PICK chart como apresentado na Figura 3. Assim, dentre as propostas apresentadas, foi selecionada a Alternativa B (fácil de implementar / alto retorno). 


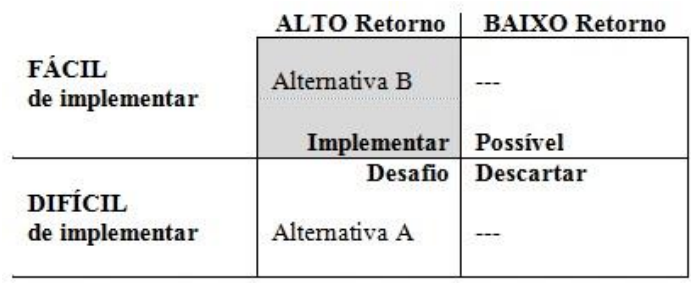

Figura 3. Classificação das alternativas de solução

As duas alternativas de solução requerem treinamento no modelo MPS-SW ou contratação de consultoria especializada, pois os analistas de qualidade da empresa ainda não dominam todos os aspectos dos novos processos a serem definidos na DMS. Além disso, é necessário o desenvolvimento ou aquisição de ferramenta para GRU.

Devido às restrições de negócio, é esperado que seja feita uma avaliação formal para o modelo CMMI-DEV ao final do $2^{\circ}$. ciclo de melhoria. Porém, após o $3^{\circ}$. ciclo é possível que avaliação seja feita nos dois modelos, favorecendo o aumento da visibilidade da empresa no mercado. Portanto, recomendou-se uma avaliação conjunta.

Atividade 4 - Definir medidas de sucesso do Programa de Melhoria: Nesta atividade são definidas as medidas relevantes para o monitoramento e avaliação dos ganhos obtidos com o programa de melhoria. Assim, para cada QC de benefício são definidas uma ou mais medidas. No Quadro 6 são apresentados alguns exemplos:

Quadro 6 - Exemplos de medidas para avaliação do retorno do investimento

\begin{tabular}{|ll}
\hline B16 - Aumento da satisfação do cliente & Medida: Grau de satisfação do cliente \\
B10 - Aumento da satisfação dos colaboradores & Medida: Grau de satisfação dos colaboradores \\
B07 - Redução de retrabalho & Medida: Taxa de retrabalho \\
B22 - Cumprimento de prazo & Medida: Desvio de prazo \\
B28 - Redução de multas & Medida: \% multas (em relação ao valor do contrato) \\
& Medida: Custo das multas pagas \\
\hline
\end{tabular}

\subsection{Empresa B}

A Empresa B está há 19 anos no mercado, tem menos de dez colaboradores, presta serviços de desenvolvimento e manutenção de software, Testes de software, desenvolvimento e comercialização de produto (COTS) e serviços de help-desk. Ela tem clientes no ramo privado, no governo e no exterior. Quanto à melhoria de processos, já foi avaliada no MPS-SW nível G, mas atualmente apenas o processo GPR é executado (parcialmente). A Estratégia foi aplicada de forma semelhante o que à aplicação feita na Empresa A, e explicaremos resumidamente os resultados obtidos.

Na Empresa B também seria aplicável uma solução multimodelo com: MPSSW; MPS-SV; CMMI-DEV; e MPT.Br. A alternativa de solução mais adequada para o $1^{\circ}$. ciclo de melhoria seria a que focasse principalmente em processos elementares como GPR e GRE, pois eles constituem uma base para os demais processos. Além disso, justamente porque alta direção foca no crescimento da empresa, é o momento de tratar de processos críticos de apoio como MED que, por sua vez, juntamente com GPP, trará conhecimento sobre a capacidade produtiva da empresa.

\subsection{Lições aprendidas e evolução da Estratégia}

As principais lições aprendidas e as oportunidades de melhoria são listadas a seguir: 
\#1 - A Estratégia foi aplicada em uma grande empresa e em uma microempresa do mercado privado. Observou-se que a obtenção de uma visão holística não é trivial, pois, independentemente do seu tamanho em número de colaboradores, as empresas podem ter multiplicidade de linhas de negócios ou de necessidades de melhoria. Por exemplo:

- Na Empresa B, foram identificados 4 modelos candidatos, embora ela seja uma microempresa. Neste caso a visão holística abrangeu a empresa toda, tanto em linhas de negócios, quanto em visões dos seus níveis hierárquicos.

- A Empresa A é uma grande empresa com várias linhas de negócios. A abrangência do estudo se deu apenas em uma linha de negócio, perpassando-a por todos os níveis hierárquicos. Assim, embora não tenha sido abordada toda a empresa, o termo holístico é aplicável por elicitar QC que perpassam todos os níveis hierárquicos.

De forma mais ampla observa-se que a visão holística pode se dar tanto em uma dimensão horizontal, perpassando várias linhas de negócios afins, quanto em uma dimensão vertical perpassando apenas uma linha de negócio, mas considerando as visões de vários níveis hierárquicos. A possibilidade de abranger essas duas dimensões depende da estrutura da empresa e do seu próprio desejo em abordá-las.

\#2 - Quanto maior o conhecimento da empresa a respeito dos seus objetivos de negócio e dos seus processos internos, maior a clareza que ela tem ao responder os questionários que, desta forma, conduzem à QC mais conscientes. Neste sentido, a função de garantia da qualidade parece ser fundamental para se ter maior consciência ao indicar as QC (este é o caso da Empresa A). Já a Empresa B, que não a possui, praticamente quase tudo que foi abordado nos questionários foi considerado crítico.

\#3 - Empresas que não possuem clareza dos seus objetivos de negócios se beneficiam desta Estratégia na medida em que as QC propiciam raciocínios sobre eles.

\#4 - Quanto à definição de medidas para avaliar os ganhos com o programa de melhoria, é importante antes aproveitar os dados que já vêm sendo coletados ao longo do tempo, de forma a minimizar esforços com a inclusão de novas medições. Este é o caso da Empresa A que possui uma base de medidas.

\#5 - Embora sejam usados alguns critérios para definição das alternativas de solução, é possível que uma nova alternativa seja definida ao discuti-las com a empresa, pois informações relevantes podem surgir nesse momento. O uso do PICK chart contribui para sistematizar a seleção da melhor alternativa. - Melhoria: estabelecer um conjunto de critérios para apoiar a empresa a melhor entender o que pode impactar na complexidade de implementação.

\#6 - Todos os colaboradores respondem todas as questões dos questionários, porém algumas delas não dizem respeito às atividades relacionadas ao seu trabalho, ou eles nem mesmo são diretamente impactados pelos resultados da execução dessas atividades. Ao fazer a consolidação, isso dificulta conclusões sobre a importância e percepção, pois quem as executa, quem é afetado por elas ou qualquer outro que não tem relação com essas atividades passam a ter o mesmo peso na indicação de QC. - Melhoria: alterar os questionários para que o respondente possa indicar se ele executa ou se é impactado pela atividade. Isso contribuirá para melhorar o processo de consolidação dos dados para seleção das QC. 
\#7 - Os questionários não possuem questões específicas para os processos do primeiro nível de maturidade. Isso implica em automaticamente recomendar à empresa a implementação de todos os processos dos níveis mais baixos, ainda que eles não sejam relevantes naquele momento. - Melhoria: alterar os questionários para incluir questões relacionadas aos processos do primeiro nível de maturidade.

\#8 - Algumas vezes a empresa pode ter interesse apenas em alguns aspectos do processo, o que implica em implementar apenas esses aspectos evitando implementar todo o processo. - Melhoria: Atualizar os questionários para endereçar os diferentes aspetos que são tratados nos processos (quando aplicável). E também estabelecer um mecanismo que apoie a empresa a selecionar os aspectos que poderiam ser excluídos ou não, a depender das suas necessidades de melhoria e também da correlação existente entre processos e até mesmo resultados esperados.

\#9 - Embora o uso do IRP contribua para explicitar os processos mais relevantes no conjunto de QC, a decisão dos processos a serem implementados nos primeiros ciclos de melhoria cabe à empresa.

\section{Considerações finais}

Este artigo apresentou a proposta de uma Estratégia para planejamento de programas de melhoria de processo em ambientes multimodelo. A seleção de modelos e processos a serem implementados é feita a partir das questões críticas apontadas pelos colaboradores nos níveis estratégico, tático e operacional. É fundamental se ter clareza dos benefícios esperados com um programa de melhoria, pois tal clareza conduz a empresa a obtê-los de forma mais consciente e abrangente. Contribuindo, portanto, para uma melhor efetividade do investimento no seu programa de melhoria. A Estratégia tem como principais vantagens: a participação dos envolvidos no programa de melhoria em diferentes níveis hierárquicos; a harmonização dos modelos que melhor se adequam às necessidades da empresa; e a visibilidade dos benefícios obtidos.

No entanto, ainda é preciso acompanhar uma execução completa da Estratégia para avaliar se as expectativas de benefícios são alcançadas. Antes disso, ainda é necessário sistematizar um pouco mais algumas atividades relacionadas com a mensuração da dificuldade de implementar uma alternativa de solução, e com a definição e seleção de alternativas de solução, embora se saiba que haverá sempre algum nível de subjetividade na tomada de decisão. Atualmente, estão sendo feitos os ajustes apontados nas lições aprendidas e está em andamento a definição do catálogo de medidas de apoio à análise de ROI em programas de melhoria.

\section{Referências}

Andelfinger, U., Heijstek, A., Kirwan, P., (2014) "A unified process improvement approach for multi-model improvement environments". In: http://www.sei.cmu.edu/library/abstracts/news-at-sei/feature1200604.cfm, access: $04 / 15$.

Birkhölzer, T., Dickmann, C., Klein, H., Vaupel, J., Ast, S., Meyer, L., (2008) "Customized predictive models for process improvement projects", v. 5089 LNCS, pp. 304-316, Monte Porzio Catone. 
Birkholzer, T., Dickmann, C., Vaupel, J., (2011) "A Framework for Systematic Evaluation of Process Improvement Priorities". In: Software Engineering and Advanced Applications (SEAA), 2011 37th EUROMICRO Conference on, pp. 294301, Aug. 30 2011-Sept. 22011.

Ferreira, A.I.F., Santos, G., Cerqueira, R., Montoni, M., Barreto, A., Rocha, A.R., Barreto, A.O.S., Silva Filho, R.C., (2008) "ROI of software process improvement at BL informática: SPIdex is really worth it", Software Process Improvement and Practice, v. 13, n. 4, pp. 311-318.

Guzmán, J.G., Mitre, H.A., Amescua, A., Velasco, M., (2010) "Integration of strategic management, process improvement and quantitative measurement for managing the competitiveness of software engineering organizations", Software Quality Journal, v. 18 , n. 3, pp. 341-359.

Ibrahim, L., (2008) "A process improvement commentary", Crosstalk: The Journal of Defense Software Engineering, v. 21, pp. 4.

Kelemen, Z.D., (2013) Process based unification for multi-model software improvement, Ritter nyomda.

Mcfeeley, B., (1996) IDEAL: A User's Guide for Software Process Improvement, CMU/SEI-96-HB-001, Pittsburgh: Software Engineering Intitute Carnegie Mellon University.

Mike, G., Rowlands, D., Castle, B., (2004) What is Lean Six Sigma?, McGraw-Hill.

Mirna, M., Jezreel, M., Jose, C.M.A., Tomas, S.F., Giner, A., (2011) "Advantages of Using a Multi-model Environment in Software Process Improvement". In: Electronics, Robotics and Automotive Mechanics Conference (CERMA), 2011 IEEE, pp. 397-402, 15-18 Nov. 2011.

Naik, K., Tripathy, P., (2008) Software testing and quality assurance, John Wiley\& Sons, Inc.

Neto, P.J.S., Abib, G., Gomel, M.M.G., Pécora, J.E., Junglos, A., Ishi, F., Braga, G., (2011) Evolução da Qualidade de Software no Brasil de 1994-2010 baseada nas pesquisas e projetos do PBQP Software, MINISTÉRIO DA CIÊNCIA, TECNOLOGIA E INOVAÇÃO, Brasília.

Parasuraman, A., Zeithaml, V., Berry, L.L., (1988) "SERVQUAL - A Multiple-Item for Scale for Measuring Consumer Perceptions of Service Quality", Journal of Retailing, v. 66 , pp. $12-40$.

Pardo, C., Pino, F., García, F., Piattini Velthius, M., Baldassarre, M., (2011) "Trends in Harmonization of Multiple Reference Models". In: MACIASZEK, L., LOUCOPOULOS, P. (eds), Evaluation of Novel Approaches to Software Engineering, Springer Berlin Heidelberg.

Pardo, C., Pino, F.J., Garcia, F., Piattini, M., Baldassarre, M.T., (2012) "An ontology for the harmonization of multiple standards and models", Computer Standards \& Interfaces, v. 34, pp. 12.

Pasquali, L., (1999) Instrumento psicológicos: manual prático de elaboração, LabPAM. 
Ramos, C.S., Oliveira, K.M., Rocha, A.R.C., (2012) "Uma Abordagem para Análise de Retorno sobre Investimentos em Programas de Melhoria de Processos de Software", pp. 44 - 53.

Ramos, C.S., Oliveira, K.M., Rocha, A.R.C., (2013) "Towards a strategy for analysing benefits of Software Process Improvement programs". In: The 25th International Conference on Software Engineering \& Knowledge Engineering, pp. 6, Boston, 27 a 29 Junho.

Ramos, C.S., Oliveira, K.M., Rocha, A.R.C., (2015) "Critical Issues in SPI Programs: A Holistic View". In: The First International Conference on Advances and Trends in Software Engineering, pp. 6, Barcelona, April.

Rico, D.F., (2004) ROI of Software Process Improvement: Metrics for Project Managers and Software Engineers, J. Ross Publishing, Inc.

Salviano, C.F., (2009) "A Multi-model Process Improvement Methodology Driven by Capability Profiles". In: COMPSAC (1), pp. 636-637.

SEI, (2010a) CMMI PRODUCT TEAM. CMMI for development. (CMU-SEI-2010-TR033). Version 1.3. Pittsburg: Software Engineering Intitute Carnegie Mellon University.

SEI, (2010b) CMMI PRODUCT TEAM. CMMI for Services. (CMU-SEI-2010-TR034). Version 1.3. Pittsburg: Software Engineering Intitute Carnegie Mellon University.

Siviy, J., Kirwan, P., Marino, L., Morley, J., (2008a) "The value of Harmonizing Multiple Improvement Technologies: A Process Improvement Professional's View". In: http://resources.sei.cmu.edu/library/asset-view.cfm?assetid=29159, access: $04 / 2015$.

Siviy, J., Kirwan, P., Morley, J., Marino, L., (2008b) "Maximizing your Process Improvement ROI through Harmonization". http://resources.sei.cmu.edu/library/asset-view.cfm?assetid=28907, access: 04/15.

Softex-Recife, (2011) MPT.Br Melhoria de Processo de Teste Brasileiro - Guia de Referência do Modelo. s.l., SOFTEX Recife.

Softex, (2012a) MPS.BR - Melhoria de Processo do Software Brasileiro - Guia Geral MPS de Serviços, Associação para Promoção da Excelência do Software Brasileiro.

Softex, (2012b) MPS.BR - Melhoria de Processo do Software Brasileiro - Guia geral MPS de Software, Associação para Promoção da Excelência do Software Brasileiro.

Softex, (2012c) MPS.BR Melhoria de Processo do Software Brasileiro - Parte 11: Implementação e Avaliação do MR-MPS-SW:2012 em Conjunto com o CMMI-DEV v1.3, Associação para Promoção da Excelência do Software Brasileiro.

Solingen, R.V., (2004) "Measuring the ROI of software process improvement", IEEE Software, v. 21, n. 3, pp. 32-38.

Xexéo, J.a.M., (2001) Sistema de informação como instrumento de programas. de qualidade, COPPE, Programa de Engenharia de Sistemas e Computação, Universidade Federal do Rio de Janeiro, Rio de Janeiro. 